\title{
CARLESON MEASURES ON SPACES OF HOMOGENEOUS TYPE
}

\author{
STEVEN C. GADBOIS AND WILLIAM T. SLEDD
}

\begin{abstract}
Let $X$ be a space of homogeneous type in the sense of Coifman and Weiss [CW2] and let $X^{+}=X \times \mathbf{R}^{+}$. A positive function $F$ on $X^{+}$is said to have horizontal bounded ratio (HBR) on $X^{+}$if there is a constant $A_{F}$ so that $F(x, t) \leq A_{F} F(y, t)$ whenever $\rho(x, y)<t$. (By Harnack's inequality, a well-known example is any positive harmonic function in the upper half plane.) HBR is a rich class that is closed under a wide variety of operations and it provides useful majorants for many classes of functions that are encountered in harmonic analysis. We are able to prove theorems in spaces of homogeneous type for functions in HBR which are analogous to the classical Carleson measure theorems and to extend these results to the functions which they majorize. These results may be applied to obtain generalizations of the original Carleson measure theorem, and of results of Flett's which contain the Hardy-Littlewood theorems on intermediate spaces of analytic functions. Hörmander's generalization of Carleson's theorem is included and it is possible to extend those results to the atomic $H^{p}$ spaces of Coifman and Weiss.
\end{abstract}

\section{INTRODUCTION}

In the course of proving the corona theorem, Carleson [Car] characterized those finite positive measures $\mu$ on the unit ball $B_{1}$ in $C^{1}$ such that

$$
\left(\int_{B_{1}}|f|^{p} d \mu\right)^{1 / p} \leq C\|f\|_{H^{p}}
$$

for every function $f$ in the Hardy space $H^{p} \quad(0<p<\infty)$, showing that this holds if and only if $\mu S \leq C^{\prime}(1-s)$ for every set $S$ of the form

$$
S=S_{s \theta_{0}}=\left\{r e^{i \theta} \mid s \leq r<1, \theta_{0}-\pi(1-s) \leq \theta<\theta_{0}+\pi(1-s)\right\} .
$$

Such a measure $\mu$ is called a Carleson measure, and such sets $S$ are called Carleson sets. The necessity of this geometric condition is easily shown by the proper choice of a function $f \in H^{p}$ which is suitably large on $S$. Carleson's proof of the sufficiency of the geometric condition used a complicated covering argument. Hörmander [Hö] derived a version for more general regions in $\mathbf{C}^{N}$ using a maximal function, Marcinkiewicz interpolation, and a simpler covering argument. Using some of Hörmander ideas, Duren [D] proved that for $0<$ $p_{1} \leq p_{2}<\infty$,

$$
\left(\int_{B_{N}}|f|^{p_{2}} d \mu\right)^{1 / p_{2}} \leq C\|f\|_{H^{p_{1}}}
$$

Received by the editors October 1, 1990 and, in revised from, January 8, 1992.

1980 Mathematics Subject Classification (1985 Revision). Primary 43A85, 42B15, 42B25, 42B30.

Key words and phrases. Carleson measure, space of homogeneous type. 
for every $f \in H^{p_{1}}$ if and only if

$$
(\mu S)^{1 / p_{2}} \leq C^{\prime}(1-s)^{1 / p_{1}}
$$

for every Carleson set $S=S_{s \theta_{0}}$.

These results concern the Hardy spaces (here, a measure on a region is compared to a measure on the boundary of the region). Other results have been obtained concerning the (weighted) Bergman spaces (here, measures on a region are compared). For example, there is a theorem due to Hastings [Ha] for the polydisc $\mathbf{D}^{N}$ (the product of $N$ copies of $\mathbf{D}=B_{1}$ ): letting $m$ denote Haar measure on $\mathbf{D}^{N}$, we have

$$
\left(\int_{\mathbf{D}^{N}}|f|^{p_{2}} d \mu\right)^{1 / p_{2}} \leq C\left(\int_{\mathbf{D}^{N}}|f|^{p_{1}} d m\right)^{1 / p_{1}}
$$

for every analytic function $f$ on $\mathbf{D}^{N}\left(0<p_{1} \leq p_{2}<\infty\right)$ if and only if

$$
(\mu S)^{1 / p_{2}} \leq C^{\prime}\left[\left(1-s_{1}\right) \cdots\left(1-s_{N}\right)\right]^{2 / p_{1}}
$$

for every set $S$ of the form $S=S_{S_{1} \theta_{01}} \times \cdots \times S_{S_{N} \theta_{0 N}}$. We will refer to any such result as a Carleson measure theorem. Cima and Wogen [CiWo] proved a Carleson measure theorem for weighted Bergman spaces in the unit ball $B_{N}$ in $\mathbf{C}^{N}$; the Cima-Wogen theorem is a consequence of Luecking's general technique [L].

The result of Duren mentioned earlier intersects some results of Hardy and Littlewood [HL2]: if $0<p_{1}<p_{2}<\infty, \alpha=1 / p_{1}-1 / p_{2}$, and $p_{1} \leq q_{2}$, then

$$
\left[\int_{0}^{1}\left(\int_{0}^{2 \pi}\left|f\left(r e^{i \theta}\right)\right|^{p_{2}} d \theta\right)^{q_{2} / p_{2}}(1-r)^{q_{2} \alpha-1} d r\right]^{1 / q_{2}} \leq C\|f\|_{H^{p_{1}}}
$$

for every $f \in H^{p_{1}}$. When $q_{2}=p_{2}$, by letting $d \mu\left(r e^{i \theta}\right)=d \theta(1-r)^{q_{2} \alpha-1} d r$ we see that this is a consequence of (1.1). When $q_{2} \neq p_{2}$, the expression on the left-hand side of (1.2) is an example of a mixed norm [BP].

Inequalities of the type found in (1.2) have been extended to more general settings by Flett [F1]. (See also [Gr].)

It is the purpose of this paper to examine conditions on measures which generalize the Carleson measure theorems stated above to the context of spaces of homogeneous type in the sense of Coifman and Weiss [CW2], and to apply those results to several interesting examples of such spaces.

In $\S 2$, the definition of a space of homogeneous type is given. This leads to a collection of operators determined by positive kernels whose shape depends on the quasi-metric and the doubling measure on the space. The classes of functions of bounded ratio and horizontal bounded ratio are introduced. Then several maximal functions analogous to those that have proved useful in other situations are defined and some basic lemmas are stated.

Section 3 is concerned with Carleson measure theorems for nontangential maximal functions. These results are applied in $\S 4$ to obtain Carleson measure theorems for maximal operators which include that used by Hörmander [Hö].

In $\S 5$ we consider several examples of spaces of homogeneous type and examine some particular operators (the Poisson and Cauchy transforms). The results of $\S 4$ may be applied to these. 
Section 6 contains results about multiplier theorems for some of the example spaces of $\S 5$, and in $\S 7$, the question of necessary conditions for Carleson measures is briefly examined.

Regarding notation, we write $f \sim g$ if $f / g$ lies between two positive constants for all values of the variables on which $f$ and $g$ depend. Constants will usually be denoted by $C$, and may vary from line to line. If $C$ depends on parameters $a, b, \ldots$, we may write $C=C(a, b, \ldots)$. The end of a proof will be denoted by

\section{SPACES OF hOMOGENEOUS TYPE AND THE CLASS BR}

We first summarize known results about spaces of homogeneous type and introduce the functions of bounded ratio and horizontal bounded ratio. Good references for spaces of homogeneous type are [CW1, CW2]. We begin with a number of definitions.

A quasi-metric on a set $X$ is a nonnegative function $\rho$ defined on $X \times X$ that satisfies $\rho(x, y)=0$ if and only if $x=y, \rho(x, y)=\rho(y, x)$ for each $x, y \in X$, and there is some constant $C$ such that

$$
\rho(x, y) \leq C[\rho(x, z)+\rho(z, y)]
$$

for each $x, y, z \in X$. The smallest such constant $C$ is denoted by $C_{\rho}$. Note that $C_{\rho} \geq 1$. Let $B(x, r)=\{y \in X \mid \rho(x, y)<r\}$; such a set is called a quasi-ball with center at $x$.

A set function $\sigma$ defined on the quasi-balls is said to be doubling if there is a constant $C$ such that

$$
0<\sigma(B(x, 2 r)) \leq C \sigma(B(x, r))
$$

for every $x \in X$ and $r>0$. The smallest such constant $C$ is denoted by $C_{\sigma}$ and is called the doubling constant for $\sigma$.

A quasi-metric space $X$ is a Hausdorff topological space on which there is defined a quasi-metric $\rho$ so that for each $x \in X$ the quasi-balls $B(x, r)$ form a base for the open sets at $x$. In addition, $X$ is said to be a space of homogeneous type if for each $C>0$ there is a number $L=L(C)$ so that if $B\left(x_{1}, r\right), \ldots, B\left(x_{k}, r\right)$ are disjoint subsets of $B(x, C r)$, then $k \leq L$ [CW1]. If $X$ is a quasi-metric space, and if there is Borel measure $\sigma$ on $X$ that is doubling then it follows that $X$ is a space of homogeneous type [CW1, p. 67].

In the sequel we will assume that $(X, \rho, \sigma)$ is a space of homogeneous type determined by a quasi-metric $\rho$ and a doubling measure $\sigma$. This is, in fact, the definition of a space of homogeneous type that is given in [CW2]. The constants $C_{\rho}$ and $C_{\sigma}$ are called the constants of $X$.

The following notation will be used. Let $X^{+}=X \times \mathbf{R}^{+}=\{(x, r) \mid x \in$ $X, r \geq 0\}$. If $B=B(x, r)$, let $\widetilde{B}=B(x, 2 r), B^{\bullet}=B\left(x, 3 C_{\rho}^{2} r\right), T(B)=$ $\{(y, s) \mid \rho(x, y)+s<r\}$, and $I_{B}=[0,2 r]$. Note that $T(B) \subset B \times I_{B} \subset T\left(B^{\bullet}\right)$. Although the Carleson set in $\mathbb{C}^{1}$ is $B \times I_{B}, T(B)$ will be more useful in what follows.

A positive function $F$ on $X^{+}$is said to be of bounded ratio if there is a constant $A_{F}$ such that for every $z \in X$ and $t>0, F(x, r) \leq A_{F}(y, s)$ whenever $(x, r),(y, s) \in B(z, t) \times[t, 2 t]$. Denote the collection of all such functions by BR. A positive function $F$ on $X^{+}$is said to be of horizontal bounded ratio if there is a constant $A_{F}$ such that for every $t>0, F(x, t) \leq$ 
$A_{F} F(y, t)$ whenever $\rho(x, y)<t$. Denote the collection of all such functions by $\mathrm{HBR}$.

Note that BR is contained in HBR. Most of the examples we will examine will be in BR, but most of the applications will concern HBR. The classes BR and HBR are both stable under most operations. In particular they are closed under addition, multiplication by positive scalars, lattice operations, and raising to any power, positive or negative. By Harnack's inequality, a familiar example of a function in $\mathrm{BR}$ is a positive harmonic function in the upper half plane in $\mathbf{R}^{2}$. In this case the sets $B(x, r) \times[r, 2 r]$ are roughly the same as the pseudo-hyperbolic balls and it is useful to think of them in this sense.

In general, for fixed $w \in X$, the function $F_{w}$ defined by $F_{w}(x, r)=$ $\rho(x, w)+r$ is in BR, a fact which will be exploited often. Another useful example is the function $G$ defined by $G(x, r)=\sigma(B(x, r))$. There are many more interesting examples of functions in BR. We now construct some of them.

Let

$$
C_{k}=\sup _{(x, r) \in X^{+}} \frac{\sigma\left(B\left(x, 2^{k} r\right)\right)}{\sigma(B(x, r))} .
$$

Note that $C_{k} \leq C_{\sigma}^{k}$.

An admissible function is a function $\Phi: \mathbf{R}^{+} \rightarrow[0,1]$ satisfying

1 (a) $\Phi(0)=1$ and $\Phi(1)>0$,

1 (b) $\Phi$ is monotone decreasing, and

1(c) $\sum_{k} C_{k} \Phi\left(2^{k}\right)<\infty$.

It follows directly from the properties of an admissible function that

$$
\begin{aligned}
\Phi(1) \sigma(B(x, t)) & \leq \int_{X} \Phi(\rho(x, z) / t) d \sigma(z) \\
& \leq \sigma(B(x, t))+\sum_{k=0}^{\infty} \Phi\left(2^{k}\right) \sigma\left(B\left(x, 2^{k+1} t\right)\right) \\
& \leq C \sigma(B(x, t))
\end{aligned}
$$

uniformly in $x$ and $t$, so that if we define

$$
K_{t}(x, y)=\frac{\Phi(\rho(x, y) / t)}{\int_{X} \Phi(\rho(x, z) / t) d \sigma(z)},
$$

then

2(a) $\int_{X} K_{t}(x, y) d \sigma(y)=1$ for each $x$ and $t$,

2(b) $K_{t}(x, y) \geq 0$ for each $x, y$, and $t$, and

2(c) $K_{t}(x, y) \sim \Phi(\rho(x, y) / t) / \sigma(B(x, t))$ uniformly in $x, y$, and $t$.

It also follows from $1(\mathrm{c})$ that

2(d) $\lim _{t \rightarrow 0} \sup _{x \in X} \int_{\{\rho(x, y) \geq \varepsilon\}} K_{t}(x, y) d \sigma(y)=0$ for each $\varepsilon>0$.

From 2(a) and 2(b) it follows that if we write

$$
\mathscr{K} g(x, t)=\int_{X} K_{t}(x, y) g(y) d \sigma(y),
$$

then for $1 \leq p \leq \infty,\|\mathscr{K} g(\cdot, t)\|_{p} \leq\|g\|_{p}$ for every $g \in L^{p}(\sigma)$. Furthermore, letting

$$
\mathscr{H} g(x, t)=\sup _{x \in B, \operatorname{rad} B>t} \frac{\int_{B}|g| d \sigma}{\sigma(B)}
$$


and

$$
\mathscr{M} g(x)=\sup _{x \in B} \frac{\int_{B}|g| d \sigma}{\sigma(B)},
$$

it is straightforward to show that $|\mathscr{K} g(x, t)| \leq C \mathscr{H} g(x, t) \leq C \mathscr{M} g(x)$. The maximal function $\mathscr{H} g$ is essentially the one introduced by Hörmander [Hö] and the maximal function $\mathscr{M} g$ is the Hardy-Littlewood maximal function relative to $\sigma$ [S]. Since $\sigma$ is a doubling measure, it is well known that $\mathscr{M}$ is weak-type $(1,1)$ and strong type $(p, p)$ for each $1<p \leq \infty$. (See [S].)

More generally, if $\nu$ is a Borel measure on $X^{+}$with $\nu(T(B))>0$ for each quasi-ball $B$, let

$$
\mathscr{H}_{\nu} f(x, t)=\sup _{(x, t) \in T(B)} \frac{\int_{T(B)}|f| d \nu}{\nu(T(B))} .
$$

Such maximal functions have been considered by Békollé [B]. Note that $\mathscr{H}_{\nu}=$ $\mathscr{H}$ for $\nu(E)=\sigma(E \cap(X \times\{0\}))$.

For $\alpha>0$, let $\Gamma_{\alpha}(x, r)=\{(y, s) \mid \rho(x, y)<\alpha(s-r)\}$, an "upward pointing cone" with vertex at $(x, r)$. Note that $(y, s) \in \Gamma_{1}(x, r)$ if and only if $(x, r) \in$ $T(B(y, s))$. For $F: X^{+} \rightarrow \mathbf{R}^{+}$, define the two maximal functions $F^{*}$ and $\mathscr{N}_{\alpha} F$ on $X^{+}$by

$$
F^{*}(x, r)=\sup _{s \geq r} F(x, s)
$$

and

$$
\mathscr{N}_{\alpha} F(x, r)=\sup _{(y, s) \in \Gamma_{\alpha}(x, r)} F(y, s) .
$$

For each $\lambda>0,\left\{\mathscr{N}_{\alpha} F>\lambda\right\}$ is open in $X^{+}$, and for each $t \geq 0$ and each $\lambda>0,\left\{\mathscr{N}_{\alpha} F(\cdot, t)>\lambda\right\}$ is open in $X$. Moreover, $\left(\mathscr{N}_{\alpha} F\right)^{*}=\mathscr{N}_{\alpha} F$ and $\left(\mathscr{N}_{\alpha} F\right)^{p}=\mathscr{N}_{\alpha}\left(F^{p}\right)$. Also note that $\mathscr{N}_{\alpha} F \leq C F^{*}$ for $F \in \mathrm{HBR}$, and $F \leq \mathscr{N}_{\alpha} F$ for lower semicontinuous $F$.

When $X=\mathbf{R}^{d}$ and $\rho(x, y)=|x-y|, \mathscr{N}_{\alpha} F$ is the usual nontangential maximal function on $X$. When $X=\mathbf{R}^{d}$ and $\rho(x, y)=|x-y|^{2}, \mathscr{N}_{\alpha} F$ is the maximal function used by Bañuelos and Moore [BM] in their study of solutions of the heat equation.

The following facts will prove to be useful in what follows.

Lemma 2.1. Suppose that $\Phi$ is an admissible function and that there is a constant $C$ such that $\Phi(t) \leq C \Phi(2 t)$ for every $t \in \mathbf{R}^{+}$.

(a) If $w \in X$ is fixed and if $f_{w}(x, r)=\Phi(\rho(x, w) / r)$, then $f_{w} \in \mathrm{BR}$, and there is a constant $C^{\prime}=C^{\prime}\left(C, C_{\rho}, \Phi\right)$ such that $A_{f_{w}} \leq C^{\prime}$ for each $w$.

(b) If $w \in X$ is fixed and if $F_{w}(x, r)=K_{r}(x, w)$, then $F_{w} \in \mathrm{BR}$, and there is a constant $C^{\prime}=C^{\prime}\left(C, C_{\rho}, \Phi\right)$ such that $A_{F_{w}} \leq C^{\prime}$ for each $w$.

(c) If $f$ is a nonnegative Borel measurable function on $X$ and if $F(x, r)=$ $\mathscr{K} f(x, r)$, then $F \in \mathrm{BR}$.

(d) If $f$ is a Borel measurable function on $X^{+}$, if $0<\nu(T(\widetilde{B})) \leq C^{\prime} \nu(T(B))$ for each quasi-ball $B$, and if $F(x, r)=\mathscr{H}_{\nu} f(x, r)$, then $F \in \mathrm{BR}$.

(e) If $F \in \mathrm{BR}$ and if $F_{r}$ defined for $r>0$ by $F_{r}(x)=F(x, r)$ is Borel measurable, then $F(x, 2 r) \leq A_{F} C_{\sigma} \mathscr{H} F_{r}(x, r)$ for each $x$ and $r$.

Proof. Fix an integer $k=k\left(C_{\rho}\right)$ satisfying $2 C_{\rho}^{2}+C_{\rho}+2 \leq 2^{k}$. 
To prove (a), fix $(z, t) \in X^{+}$and suppose that $(x, r),(y, s) \in B(z, t) \times$ $[t, 2 t]$ (so $\left.\rho(x, y)<2 C_{\rho} t \leq 2 C_{\rho} s\right)$. Then

$$
\begin{aligned}
f_{w}(x, r) & =\Phi(\rho(x, w) / r) \leq \Phi(\rho(x, w) / 2 t) \\
& \leq \Phi(\rho(x, w) / 2 s) \leq C \Phi(\rho(x, w) / s) .
\end{aligned}
$$

We now consider three cases. First, if $\rho(x, w) \geq \rho(y, w)$, then $\Phi(\rho(x, w) / s)$ $\leq \Phi(\rho(y, w) / s)$, hence $f_{w}(x, r) \leq C f_{w}(y, s)$. Second, if $s \leq \rho(x, w) \leq$ $\rho(y, w)$, then

$$
\begin{aligned}
\rho(y, w) & \leq C_{\rho}[\rho(x, y)+\rho(x, w)]<C_{\rho}\left[2 C_{\rho} s+\rho(x, w)\right] \\
& \leq C_{\rho}\left(2 C_{\rho}+1\right) \rho(x, w)<2^{k} \rho(x, w),
\end{aligned}
$$

so $\Phi(\rho(x, w) / s) \leq C^{k} \Phi(\rho(y, w) / s)$, hence $f_{w}(x, r) \leq C^{k+1} f_{w}(y, s)$. Third, if $\rho(x, w) \leq s$ and $\rho(x, w) \leq \rho(y, w)$, then

$$
\rho(y, w) \leq C_{\rho}[\rho(x, y)+\rho(x, w)]<C_{\rho}\left(2 C_{\rho}+1\right) s<2^{k} s,
$$

so

$$
\Phi(\rho(x, w) / s) \leq \Phi(0)=1 \leq \frac{C^{k}}{\Phi(1)} \Phi\left(2^{k}\right) \leq \frac{C^{k}}{\Phi(1)} \Phi(\rho(y, w) / s),
$$

hence $f_{w}(x, r) \leq C^{k+1} f_{w}(y, s) / \Phi(1)$.

Statement (b) is an immediate consequence of (a). Fix $(z, t) \in X^{+}$and suppose that $(x, r),(y, s) \in B(z, t) \times[t, 2 t]$. Then

$$
\begin{aligned}
F_{w}(x, r) & =K_{r}(x, w)=\frac{\Phi(\rho(x, w) / r)}{\int_{X} \Phi(\rho(x, z) / r) d \sigma(z)}=\frac{f_{w}(x, r)}{\int_{X} f_{z}(x, r) d \sigma(z)} \\
& \leq \frac{C^{\prime} f_{w}(y, s)}{\int_{X}\left(C^{\prime}\right)^{-1} f_{z}(y, s) d \sigma(z)}=\left(C^{\prime}\right)^{2} F_{w}(y, s) .
\end{aligned}
$$

We prove (c) using (b). Fix $(z, t) \in X^{+}$and suppose that $(x, r),(y, s) \in$ $B(z, t) \times[t, 2 t]$. Then

$$
\begin{aligned}
F(x, r) & =\mathscr{K} f(x, r)=\int_{X} K_{r}(x, w) f(w) d \sigma(w) \\
& =\int_{X} F_{w}(x, r) f(w) d \sigma(w) \leq \int_{X} A_{F_{w}} F_{w}(y, s) f(w) d \sigma(w) \\
& \leq \int_{X} C^{\prime} F_{w}(y, s) f(w) d \sigma(w)=C^{\prime} F(y, s) .
\end{aligned}
$$

To prove (d), fix $(z, t) \in X^{+}$and suppose that $(x, r),(y, s) \in B(z, t) \times$ $[t, 2 t]$ (so $\left.\rho(x, y)<2 C_{\rho} t\right)$. Suppose that $B_{0}=B_{0}\left(z_{0}, t_{0}\right)$ satisfies $(x, r) \in$ $T\left(B_{0}\right)$, i.e., $\rho\left(z_{0}, x\right)+r<t_{0}$. Then

$$
\begin{aligned}
\rho\left(z_{0}, y\right)+s & \leq C_{\rho}\left[\rho\left(z_{0}, x\right)+\rho(x, y)\right]+s<C_{\rho}\left[t_{0}+2 C_{\rho} t\right]+s \\
& <\left(2 C_{\rho}^{2}+C_{\rho}+2\right) t_{0} \leq 2^{k} t_{0},
\end{aligned}
$$

so that $B_{k}=B_{k}\left(z_{0}, 2^{k} t_{0}\right)$ satisfies $(y, s) \in T\left(B_{k}\right)$, and thus

$$
\frac{\int_{T\left(B_{0}\right)}|f| d \nu}{\nu\left(T\left(B_{0}\right)\right)} \leq\left(C^{\prime}\right)^{k} \frac{\int_{T\left(B_{k}\right)}|f| d \nu}{\nu\left(T\left(B_{k}\right)\right)} \leq\left(C^{\prime}\right)^{k} F(y, s) .
$$

The result follows. 
Finally, to prove (e), for every $y \in B=B(x, r), F(x, 2 r) \leq A_{F} F(y, r)=$ $A_{F} F_{r}(y)$, and thus

$$
F(x, 2 r) \leq A_{F} \frac{\int_{B} F_{r} d \sigma}{\sigma(B)} \leq A_{F} \frac{\int_{\widetilde{B}} F_{r} d \sigma}{\sigma(\widetilde{B}) / C_{\sigma}} \leq A_{F} C_{\sigma} \mathscr{H} F_{r}(x, r) .
$$

The following "covering lemma" contains some known facts about spaces of homogeneous type.

Lemma 2.2. (a) If $\mathscr{B}$ is a family of quasi-balls with bounded radius, then there is a countable disjoint family of quasi-balls $\left\{B_{n}\right\}$ contained in $\mathscr{B}$ so that for each $B \in \mathscr{B}$, there is a $B_{n}$ so that $B \subset B_{n}^{\cdot}$.

(b) For each $r>0$, there is a countable family of quasi-balls $\left\{B\left(x_{n}, r\right)\right\}$ so that the family $\left\{B\left(x_{n}, r / 2 C_{\rho}\right)\right\}$ is disjoint, $X=\bigcup_{n} B\left(x_{n}, r\right)$, and the family is maximal with respect to this property.

(c) There is a constant $L=L\left(C_{\rho}\right)$, independent of $r$, so that $\sum_{n} \chi_{B\left(x_{n}, r\right)} \leq L$ on $X$.

Proof. Statement (a) is Lemma 3 in [Cal], and (b) is implicit in that proof. Finally, (c) follows from the maximality of the family since $X$ is a space of homogeneous type.

\section{CARleson MEASURE THEOREMS FOR NONTANGENTIAL MAXIMAL FUNCTIONS}

We shall say that a function $\mu$ defined on the Borel sets of $X^{+}$satisfies condition $H$ if $\mu$ is nonnegative, countably subadditive, and $\mu\left(E_{n}\right) \rightarrow \mu(E)$ whenever $\left\{E_{n}\right\}$ is a sequence of Borel sets with $E_{n} \subseteq E_{n+1}$ and $E=\bigcup_{n} E_{n}$. These conditions on $\mu$ are quite similar to those assumed by Hörmander [Hö]. Note that they are satisfied whenever $\mu$ is a Borel measure or a capacity [Fu]. The utility of such generalization will be seen in Corollary 3.2. The proof of Theorem 3.1 is similar to the proof of the Carleson measure theorem due to Stein [S].

Theorem 3.1. (a) Suppose that $\mu$ satisfies condition $H$, that $\nu$ is a Borel measure on $X^{+}$, and that

$$
\mu\left(T\left(\widetilde{B}^{\bullet}\right)\right) \leq \nu(T(B))
$$

for every quasi-ball $B$. Then $\mu\left(\left\{\mathscr{N}_{1} F>\lambda\right\}\right) \leq \nu\left(\left\{\mathscr{N}_{C_{\rho}} F>\lambda\right\}\right)$ for every $\lambda>0$.

(b) Suppose that $\mu$ satisfies condition $H$ and that $\varphi$ defined by

$$
\phi(x)=\sup _{x \in B} \frac{\mu(T(B))}{\sigma(B)}
$$

is locally integrable with respect to $\sigma$, and let $d \nu=\phi d \sigma$. Then there is a constant $C=C\left(C_{\rho}\right)$ such that $\mu\left(\left\{\mathscr{N}_{1} F>\lambda\right\}\right) \leq C \nu\left(\left\{\mathscr{N}_{C_{\rho}} F(\cdot, 0)>\lambda\right\}\right)$ for every $\lambda>0$.

Proof. To prove (a), fix $b, c>0$, set $G(x, r)=\min \left\{b, \mathscr{N}_{1} F(x, r)\right\} /(1+c r)$, and suppose that $G(x, r)>\lambda$. Then $r<b / c \lambda$ and $\mathscr{N}_{1} F(x, r)>\lambda$. So if $\mathscr{B}=\{B(x, r) \mid G(x, r)>\lambda\}$ then from Lemma 2.2(a), there is a countable disjoint subfamily $\left\{B_{n}\right\}$ of $\mathscr{B}$ such that each $B$ in $\mathscr{B}$ is in some $B_{n}^{\bullet}$. Then $\{G>\lambda\} \subset \bigcup_{n} T\left(\widetilde{B}_{n}^{\bullet}\right)$, so

$$
\mu(\{G>\lambda\}) \leq \sum_{n} \mu\left(T\left(\widetilde{B}_{n}^{\circ}\right)\right) \leq \sum_{n} \nu\left(T\left(B_{n}\right)\right)=\nu\left(\bigcup_{n} T\left(B_{n}\right)\right) .
$$


If $B_{n}=B\left(x_{n}, r_{n}\right)$ and $(y, s) \in T\left(B_{n}\right)$, then $\left(x_{n}, r_{n}\right) \in \Gamma_{1}(y, s)$, so $\Gamma_{1}\left(x_{n}, r_{n}\right)$ $\subset \Gamma_{C_{\rho}}(y, s)$. Consequently, $\mathscr{N}_{C_{\rho}} F(y, s) \geq \mathscr{N}_{1} F\left(x_{n}, r_{n}\right)>\lambda$, and so $\bigcup_{n} T\left(B_{n}\right)$ $\subset\left\{\mathscr{N}_{C_{\rho}} F>\lambda\right\}$; thus $\mu(\{G>\lambda\}) \leq \nu\left(\left\{\mathscr{N}_{C_{\rho}} F>\lambda\right\}\right)$. To complete the proof of (a), let $b \rightarrow \infty$ and $c \rightarrow 0$ and use condition $H$.

To prove (b), first note that if $x \in B$, then

$$
\mu\left(T\left(\widetilde{B}^{\bullet}\right)\right) \leq \phi(x) \sigma\left(\widetilde{B}^{\bullet}\right) \leq C \phi(x) \sigma(B)
$$

since $\sigma$ is a doubling measure. Integrating both sides of this inequality over $B$ gives $\mu\left(T\left(\widetilde{B}^{\bullet}\right)\right) \leq C \nu(B)=C \nu^{\prime}(T(B))$, where $\nu^{\prime}(E)=\nu(E \cap(X \times\{0\}))$. It follows from (a) that

$$
\mu\left(\left\{\mathscr{N}_{1} F>\lambda\right\}\right) \leq C \nu^{\prime}\left(\left\{\mathscr{N}_{C_{\rho}} F>\lambda\right\}\right)=C \nu\left(\left\{\mathscr{N}_{C_{\rho}} F(\cdot, 0)>\lambda\right\}\right),
$$

and this completes the proof of $(b)$.

The condition $\phi(x) \leq C$ is the original condition assumed by Carleson. The more general assumption made in (b) was introduced by Fefferman and Stein [FS].

The first corollary of Theorem 3.1 is related to the result of Duren that was mentioned in the introduction.

Corollary 3.2. Let $1 \leq p<\infty$. Suppose that $\mu$ and $\nu$ are Borel measures on $X^{+}$such that $\left(\mu\left(T\left(\widetilde{B^{\bullet}}\right)\right)\right)^{1 / p} \leq \nu(T(B))$ for every quasi-ball $B$. Then there is $a$ constant $C=C(p)$ such that

$$
\left(\int_{X^{+}}\left(\mathscr{N}_{1} F\right)^{p} d \mu\right)^{1 / p} \leq C \int_{X^{+}} \mathscr{N}_{C_{\rho}} F d \nu
$$

for every nonnegative function $F$ defined on $X^{+}$.

Proof. Since $p \geq 1, \mu^{1 / p}$ also satisfies the hypotheses of the set function in Theorem 3.1. Consequently,

$$
\begin{aligned}
& \int_{X^{+}}\left(\mathscr{N}_{1} F\right)^{p} d \mu=p \int_{0}^{\infty} \lambda^{p-1} \mu\left(\left\{\mathscr{N}_{1} F>\lambda\right\}\right) d \lambda \\
& \leq C \sum_{k=-\infty}^{\infty} 2^{k p} \mu\left(\left\{\mathscr{N}_{1} F>2^{k}\right\}\right) \leq C\left(\sum_{k=-\infty}^{\infty} 2^{k}\left(\mu\left(\left\{\mathscr{N}_{1} F>2^{k}\right\}\right)\right)^{1 / p}\right)^{p} \\
& \quad \leq C\left(\sum_{k=-\infty}^{\infty} 2^{k} \nu\left(\left\{\mathscr{N}_{C_{\rho}} F>2^{k}\right\}\right)\right)^{p} \leq C\left(\int_{X^{+}} \mathscr{N}_{C_{\rho}} F d \nu\right)^{p}
\end{aligned}
$$

as claimed.

It follows immediately from Corollary 3.2 and the remarks preceding Lemma 2.1 that

$$
\left(\int_{X^{+}} F^{p} d \mu\right)^{1 / p} \leq C \int_{X^{+}} F^{*} d \nu
$$

for every lower semicontinuous $F \in$ BR. Corollary 3.3 and Theorem 3.4 will imply analogous statements for such functions $F$. Corollary 3.3 is related to the result of Flett that was mentioned in the introduction. 
Corollary 3.3. Let $1 \leq p<\infty$. Suppose that $\mu, \gamma$, and $\nu$ are Borel measures on $X, \mathbf{R}^{+}$, and $X^{+}$respectively and that

$$
\left(\mu\left(\widetilde{B}^{\bullet}\right)\right)^{1 / p} \gamma\left(I_{\widetilde{B}^{\bullet}}\right) \leq \nu(T(B))
$$

for every quasi-ball $B$. Then

$$
\int_{\mathbf{R}^{+}}\left(\int_{X}\left(\mathscr{N}_{1} F\right)^{p} d \mu\right)^{1 / p} d \gamma \leq \int_{X^{+}} \mathscr{N}_{C_{\rho}} F d \nu
$$

Proof. Assume that $p>1$, since the case $p=1$ is covered by Corollary 3.2. There is a function $G$ defined on $X^{+}$such that for each $r \geq 0$,

$$
\left(\int_{X}\left(\mathscr{N}_{1} F(x, r)\right)^{p} d \mu(x)\right)^{1 / p}=\int_{X} \mathscr{N}_{1} F(x, r) G(x, r) d \mu(x)
$$

and $\int_{X} G^{p^{\prime}}(x, r) d \mu(x)=1$, where $p^{\prime}$ is the index conjugate to $p$. Let $d \beta=$ $G d \mu d \gamma$. Then $\beta\left(T\left(\widetilde{B}^{\bullet}\right)\right) \leq\left(\mu\left(\widetilde{B}^{\bullet}\right)\right)^{1 / p} \gamma\left(I_{\widetilde{B}^{\bullet}}\right) \leq \nu(T(B))$ for every $x \in B$. So from Theorem 3.1,

$$
\int_{\mathbf{R}^{+}}\left(\int_{X}\left(\mathscr{N}_{1} F\right)^{p} d \mu\right)^{1 / p} d \gamma=\int_{X^{+}} \mathscr{N}_{1} F d \beta \leq \int_{X^{+}} \mathscr{N}_{C_{\rho}} F d \nu
$$

as desired.

The next result deals with inequalities involving mixed norm spaces on both sides. It does not require a covering argument of the type that was used in Theorem 3.1.

Theorem 3.4. Let $\alpha, \kappa \geq 1$ and $\beta>0$. Suppose that $\mu, \lambda, \nu$, and $\gamma$ are Borel measures on $X, \mathbf{R}^{+}, X$, and $\mathbf{R}^{+}$respectively and that

$$
(\mu(B(x, r / 2)))^{\beta} \lambda[r, 2 r] \leq(\nu(B(x, r / 4)))^{\alpha \beta}(\gamma[r / 8, r / 4])^{\kappa}
$$

for each $x \in X$ and $r \geq 0$. Then there is a constant $C=C\left(C_{\rho}, \alpha, \beta\right)$ such that

$$
\int_{\mathbf{R}^{+}}\left(\int_{X}\left(\mathscr{N}_{1} F\right)^{\alpha} d \mu\right)^{\beta} d \lambda \leq C\left[\int_{\mathbf{R}^{+}}\left(\int_{X} \mathscr{N}_{C_{\rho}^{2}} F d \nu\right)^{\alpha \beta / \kappa} d \gamma\right]^{\kappa}
$$

for every nonnegative function $F$ defined on $X^{+}$.

Proof. For each integer $n$, let $J_{n}=\left(2^{-n-1}, 2^{-n}\right.$, and let $L$ be the constant and $\left\{B_{n k}\right\}_{k}$ the family of balls of radius $2^{-n-2}$ whose existence and properties are guaranteed by Lemma 2.2. Let $c_{n k}$ denote the center of $B_{n k}$. Note that if $(x, r) \in B_{n k} \times J_{n}$, then $\Gamma_{1}(x, r) \subset \Gamma_{C_{\rho}}\left(c_{n k}, 2^{-n-2}\right)$, so that $\mathscr{N}_{1} F(x, t) \leq \mathscr{N}_{C_{p}} F\left(c_{n k}, 2^{-n-2}\right)$. Also note that if $x \in B\left(c_{n k}, 2^{-n-3}\right)$, then 


$$
\begin{aligned}
\Gamma_{C_{\rho}}\left(c_{n k}, 2^{-n-2}\right) \subset \Gamma_{C_{\rho}^{2}}\left(x, 2^{-n-3}\right) \text {. Then } \\
\int_{\mathbf{R}^{+}}\left(\int_{X}\left(\mathscr{N}_{1} F\right)^{\alpha} d \mu\right)^{\beta} d \lambda \leq \sum_{n} \int_{J_{n}}\left(\sum_{k} \int_{B_{n k}}\left(\mathscr{N}_{1} F\right)^{\alpha} d \mu\right)^{\beta} d \lambda \\
\leq \sum_{n}\left(\sum_{k}\left(\mathscr{N}_{C_{\rho}} F\left(c_{n k}, 2^{-n-2}\right)\right)^{\alpha} \mu\left(B_{n k}\right)\right)^{\beta} \lambda\left(J_{n}\right) \\
\leq \sum_{n}\left(\sum_{k}\left(\mathscr{N}_{C_{\rho}} F\left(c_{n k}, 2^{-n-2}\right)\right)^{\alpha}\left(\nu\left(B\left(c_{n k}, 2^{-n-3}\right)\right)\right)^{\alpha}\right)^{\beta}\left(\gamma\left(J_{n+3}\right)\right)^{\kappa} \\
\leq\left[\sum_{n}\left(\sum_{k} \mathscr{N}_{C_{\rho}} F\left(c_{n k}, 2^{-n-2}\right) \nu\left(B\left(c_{n k}, 2^{-n-3}\right)\right)\right)^{\alpha \beta / \kappa} \gamma\left(J_{n+3}\right)\right]^{\kappa}(\text { since } \alpha, \kappa \geq 1) \\
\leq\left[\sum_{n}\left(\int_{X} \mathscr{N}_{C_{\rho}^{2}} F\left(x, 2^{-n-3}\right) \sum_{k} \chi_{B\left(c_{n k}, 2-n-3\right)}(x) d \nu\right)^{\alpha \beta / \kappa} \gamma\left(J_{n+3}\right)\right]^{\kappa} \\
\leq \\
\leq L^{\alpha \beta}\left[\int_{\mathbf{R}^{+}}\left(\int_{X} \mathscr{N}_{C_{\rho}^{2}} F d \nu\right)^{\alpha \beta / \kappa} d \gamma\right]^{\kappa},
\end{aligned}
$$

as claimed.

The next theorem will be useful in the next section in tying together the earlier results.

Theorem 3.5. Suppose that $\mu$ and $\nu$ are Borel measures on $X^{+}$and that $0<$ $\nu((T(B)))$ and

for every quasi-ball $B$. Then

$$
\mu\left(T\left(B^{\bullet}\right)\right) \leq \nu((T(B)))
$$

$$
\mu\left(\left\{\mathscr{H}_{\nu} f>\lambda\right\}\right) \leq \int_{X^{+}}|f| d \nu / \lambda
$$

for every $f \in L^{1}(\nu)$ and $\lambda>0$.

Proof. The proof is very much like that of Theorem 3.1. Fix $0<N<\infty$ and define

$$
\mathscr{H}_{\nu}^{N} f(x, r)=\sup _{(x, r) \in T(B), N>r} \frac{\int_{T(B)}|f| d \nu}{\nu(T(B))} .
$$

So $\mathscr{H}_{\nu}{ }^{N} f$ increases to $\mathscr{H}_{\nu} f$ as $N \rightarrow \infty$.

If $\mathscr{H}_{\nu}^{N} f(x, r)>\lambda$, then there is a quasi-ball $B$ such that $(x, r) \in T(B)$, $\operatorname{rad} B<N$, and $\nu(T(B))<\int_{T(B)}|f| d \nu / \lambda$. Let $\mathscr{B}$ denote the collection of all such balls and extract a countable disjoint subset $\left\{B_{n}\right\}$ such that $\left\{T\left(B_{n}^{\bullet}\right)\right\}$ covers $\left\{\mathscr{H}_{\nu}^{N} f>\lambda\right\}$. Then

$$
\begin{aligned}
\mu\left(\left\{\mathscr{H}_{\nu}^{N} f>\lambda\right\}\right) & \leq \sum_{n} \mu\left(T\left(B_{n}^{\bullet}\right)\right) \leq \sum_{n} \nu\left(T\left(B_{n}\right)\right) \\
& \leq \sum_{n} \int_{T\left(B_{n}\right)}|f| d \nu / \lambda \leq \int_{X^{+}}|f| d \nu / \lambda
\end{aligned}
$$

since the sets $T\left(B_{n}\right)$ are disjoint. Now let $N \rightarrow \infty$ to complete the proof. 


\section{Applications}

We now will apply the results of $\S 3$ to obtain inequalities estimating the rate of growth of rather general classes of functions by dominating them by members of HBR and using the results that we have already obtained. The choices made here by no means exhaust all of the applications.

Theorem 4.1. Let $1<p<\infty$. Suppose that $\mu$ and $\nu$ are Borel measures on $X^{+}$and that $0<\nu((T(B)))$ and

$$
\mu\left(T\left(B^{\bullet}\right)\right) \leq \nu((T(B)))
$$

for every quasi-ball $B$. Then there is a constant $C=C(p)$ such that

$$
\int_{X^{+}}\left(\mathscr{H}_{\nu} F\right)^{p} d \mu \leq C \int_{X^{+}} F^{p} d \nu
$$

for every nonnegative Borel measurable function $F$ on $X^{+}$.

Proof. This follows from Theorem 3.5 and the Marcinkiewicz interpolation theorem.

Theorem 4.2. Let $1<p \leq q<\infty$.

(a) Suppose that $\mu$ and $\nu$ are Borel measures on $X^{+}$and that $(\mu(T(B)))^{p / q} \leq$ $\nu(T(B))$ and $0<\nu(T(\widetilde{B})) \leq C \nu(T(B))$ for every quasi-ball $B$. Then there is a constant $C^{\prime}=C^{\prime}(C, p, q)$ such that

$$
\left(\int_{X^{+}}\left(\mathscr{H}_{\nu} F\right)^{q} d \mu\right)^{p / q} \leq C^{\prime} \int_{X^{+}} F^{p} d \nu
$$

for every nonnegative Borel measurable function $F$ on $X^{+}$.

(b) Suppose that $\mu, \gamma$, and $\nu$ are Borel measures on $X, \mathbf{R}^{+}$, and $X^{+}$respectively and that $0<\nu(T(\widetilde{B})) \leq C \nu(T(B))$ and $(\mu(B))^{p / q} \gamma\left(I_{B}\right) \leq \nu(T(B))$ for every quasi-ball $B$. Then there is a constant $C^{\prime}=C^{\prime}(C, p, q)$ such that

$$
\int_{\mathbf{R}^{+}}\left(\int_{X}\left(\mathscr{H}_{\nu} F\right)^{q} d \mu\right)^{p / q} d \gamma \leq C^{\prime} \int_{X^{+}} F^{p} d \nu
$$

for every nonnegative Borel measurable function $F$ on $X^{+}$.

Proof. To prove (a), note that $\left(\mathscr{H}_{\nu} F\right)^{p}$ is in BR by Lemma $2.1(\mathrm{~d})$ and is lower semicontinuous on $X^{+}$. Moreover, $\left(\mathscr{H}_{\nu} F\right)^{*}=\mathscr{H}_{\nu} F$. Consequently,

$$
\left(\mathscr{H}_{\nu} F\right)^{q} \leq\left(\mathscr{N}_{1} \mathscr{H}_{\nu} F\right)^{q}=\mathscr{N}_{1}\left(\mathscr{H}_{\nu} F\right)^{q}
$$

and

$$
\left(\mathscr{N}_{C_{\rho}}\left(\mathscr{H}_{\nu} F\right)\right)^{p} \leq C\left(\left(\mathscr{H}_{\nu} F\right)^{p}\right)^{*}=C\left(\mathscr{H}_{\nu} F\right)^{p} .
$$

So from Corollary 3.2 and Theorem 4.1 it follows that

$$
\begin{aligned}
& \left(\int_{X^{+}}\left(\mathscr{H}_{\nu} F\right)^{q} d \mu\right)^{p / q} \leq\left(\int_{X^{+}}\left(\mathscr{N}_{1}\left(\mathscr{H}_{\nu} F\right)^{p}\right)^{q / p} d \mu\right)^{p / q} \\
& \quad \leq C \int_{X^{+}} \mathscr{N}_{C_{\rho}}\left(\mathscr{H}_{\nu} F\right)^{p} d \nu \leq C \int_{X^{+}}\left(\mathscr{H}_{\nu} F\right)^{p} d \nu \leq C \int_{X^{+}} F^{p} d \nu .
\end{aligned}
$$

The proof of (b) is similar, using Corollary 3.3. 
Both Theorems 4.1 and 4.2 yield inequalities for operators determined by admissible functions, since, as we have seen earlier, these are dominated by the Hörmander operator.

Before we proceed it will be necessary to recall some definitions.

Fix $0<p \leq 1$. A $p$-atom $a$ is a Borel measurable function on $X$ whose suppose is contained in a quasi-ball $B$ and which satisfies $\int_{X} a d \sigma=0$ and $|a| \leq(\sigma(B))^{-1 / p}$. The space $H_{\text {atom }}^{1}$ is defined by

$$
H_{\text {atom }}^{1}=\left\{\sum_{n} \lambda_{n} a_{n} \mid \text { each } a_{n} \text { is a 1-atom and } \sum_{n}\left|\lambda_{n}\right|<\infty\right\},
$$

with the norm of a function $f \in H_{\text {atom }}^{1}$ defined to be the infimum of $\sum_{n}\left|\lambda_{n}\right|$ over all representations $f=\sum_{n} \lambda_{n} a_{n}$. The definition of $H_{\text {atom }}^{p}$ for $0<p<1$ is more complicated. See [CW2] for the definition of these spaces and a discussion of their applications and importance.

Recall that

$$
C_{k}=\sup _{(x, r) \in X^{+}} \frac{\sigma\left(B\left(x, 2^{k} r\right)\right)}{\sigma(B(x, r))}
$$

Theorem 4.3. Let $M$ and $m$ be positive constants and let $M /(M+m)<p \leq 1$. Suppose that $\sup _{k} C_{k} 2^{-k M}<\infty$, that $K_{t}(x, y)$ is defined for each $t \in \mathbf{R}^{+}$and each $x, y \in X$, and that $K_{t}(x, \cdot) \in L^{2}(\sigma)$ for each $t \in \mathbf{R}^{+}$and each $x \in X$. Moreover, suppose that there is a constant $C$ such that for each $z \in X$,

$$
\left|K_{t}(x, y)-K_{t}(x, z)\right| \leq C \frac{1}{\sigma(B(x, t))}\left(\frac{R}{t}\right)^{m}\left(1+\frac{\rho(x, z)}{t}\right)^{-M-m}
$$

whenever $\rho(y, z)<R$ and $\rho(x, z) \geq 2 C_{\rho} R$ and that

$$
\left\|\mathscr{N}_{\alpha}(\mathscr{K} f)\right\|_{L^{2}(\sigma)} \leq C\|f\|_{L^{2}(\sigma)}
$$

for every $f \in L^{2}(\sigma)$. Then there is a constant $C^{\prime}=C^{\prime}\left(C, C_{\rho}, M, m, p\right)$ so that

$$
\left\|\mathscr{N}_{\alpha}(\mathscr{K} a)\right\|_{L^{p}(\sigma)} \leq C^{\prime}
$$

for every $p$-atom $a$. RT].

Hypotheses of the sort imposed on $K_{t}(x, y)$ have been considered by [AB,

Proof. Let

$$
G(x, t)=\frac{1}{\sigma(B(x, t))}\left(\frac{R}{t}\right)^{m}\left(1+\frac{\rho(x, z)}{t}\right)^{-M-m} .
$$

Fix any $p$-atom $a$, so that the support of $a$ is contained in some ball $B=$ $B(z, R)$, with $|a| \leq(\sigma(B))^{-1 / p}$. Then

$$
\begin{aligned}
\int_{B\left(z, 2 C_{\rho} R\right)}\left(\mathscr{N}_{\alpha}(\mathscr{K} a)\right)^{p} d \sigma \\
\quad \leq\left(\int_{B\left(z, 2 C_{\rho} R\right)}\left(\mathscr{N}_{\alpha}(\mathscr{K} a)\right)^{2} d \sigma\right)^{p / 2}\left(\sigma\left(B\left(z, 2 C_{\rho} R\right)\right)\right)^{1-p / 2} \\
\leq C\|a\|_{2}^{p}\left(\sigma\left(B\left(z, 2 C_{\rho} R\right)\right)\right)^{1-p / 2} \leq C .
\end{aligned}
$$


Next, since $\int_{X} a d \sigma=0$,

$$
\begin{aligned}
|\mathscr{K} a(x, t)| & =\left|\int_{X}\left[K_{t}(x, y)-K_{t}(x, z)\right] a(y) d \sigma(y)\right| \\
& \leq C G(x, t) \int_{X}|a| d \sigma
\end{aligned}
$$

if $x \notin B\left(z, 2 C_{\rho} R\right)$ and so $|\mathscr{K} a(x, t)| \leq C(\sigma(B))^{1-1 / p} G(x, t)$. Note that $G$ is in BR, so $\mathscr{N}_{\alpha} G \leq A_{G} G^{*}$, and $\mathscr{N}_{\alpha}(\mathscr{K} a)(x, 0) \leq C(\sigma(B))^{1-1 / p} G^{*}(x, 0)$ whenever $\rho(x, z) \geq 2 C_{\rho} R$.

Choose $k$ so that $2^{k} C_{\rho} R \leq \rho(x, z) \leq 2^{k+1} C_{\rho} R$, so $B\left(z, 2^{k+1} C_{\rho} R\right) \subset$ $B\left(x, 2^{k+2} C_{\rho}^{2} R\right)$. We now use this to show that

$$
G(x, t) \leq C / 2^{k m} \sigma\left(B\left(z, 2^{k+1} C_{\rho} R\right)\right) .
$$

Two cases will be considered: $t \geq 2{ }^{k} C_{\rho} R$ and $t \leq 2^{k} C_{\rho} R$.

If $t \geq 2{ }^{k} C_{\rho} R$, then $B\left(z, 2^{k+1} C_{\rho} R\right) \subset B\left(x, 4 C_{\rho} t\right)$. So

$$
G(x, t) \leq C / 2^{k m} \sigma\left(B\left(z, 2^{k+1} C_{\rho} R\right)\right) .
$$

On the other hand, if $t \leq 2^{k} C_{\rho} R$, choose $l$ so that $2^{k-l-1} C_{\rho} R \leq t \leq 2^{k-l} C_{\rho} R$. Then $B\left(z, 2^{k+1} C_{\rho} R\right) \subset B\left(x, 2^{l+3} C_{\rho} t\right)$, so

$$
\sigma\left(B\left(z, 2^{k+1} C_{\rho} R\right)\right) \leq C C_{l} \sigma(B(x, t)) .
$$

Also,

$$
\left(\frac{R}{t}\right)^{m}\left(1+\frac{\rho(x, z)}{t}\right)^{-M-m} \leq\left(\frac{R}{t}\right)^{m} \frac{t^{M+m}}{(\rho(x, z))^{M+m}} \leq \frac{C}{2^{k m+l M}} .
$$

So in this case too,

$$
G(x, t) \leq \frac{C C_{l}}{2^{k m+l M} \sigma\left(B\left(z, 2^{k+1} C_{\rho} R\right)\right)} \leq \frac{C}{2^{k m} \sigma\left(B\left(z, 2^{k+1} C_{\rho} R\right)\right)} .
$$

Hence we obtain

$$
\begin{aligned}
\int_{X \backslash B\left(z, 2 C_{\rho} R\right)} G^{* p} d \sigma & =\sum_{k=1}^{\infty} \int_{\left\{2^{k} C_{\rho} R \leq \rho(x, z) \leq 2^{k+1} C_{\rho} R\right\}} G^{* p}(x) d \sigma(x) \\
& \leq C \sum_{k=1}^{\infty} 2^{-k p m} \sigma\left(B\left(z, 2^{k+1} C_{\rho} R\right)\right)^{1-p} \\
& \leq C(\sigma(B))^{1-p} \sum_{k=1}^{\infty} C_{k}^{1-p} 2^{-k p m} \leq C(\sigma(B))^{1-p}
\end{aligned}
$$

since $M /(M+m)<p \leq 1$. So $\left\|\mathcal{N}_{\alpha}(\mathscr{K} a)\right\|_{p} \leq C^{\prime}$.

The following corollaries are immediate consequences of Theorem 4.3 and Corollaries 3.2 and 3.3. In both, $\mathscr{K}$ denotes a kernel satisfying the hypotheses of Theorem 4.3.

Corollary 4.4. Let $M$ and $m$ be positive constants, let $M /(M+m)<p \leq 1$, and let $\alpha \geq 1$. Suppose that $\sup _{k} C_{k} 2^{-k M}<\infty$, that $\mu$ is a Borel measure on $X^{+}$, and that $(\mu(T(B)))^{1 / \alpha} \leq \sigma(B)$ for every quasi-ball $B$. Then there is a 
constant $C=C\left(C_{\rho}, M, m, p, \alpha\right)$ such that

$$
\int_{X^{+}}\left(\mathscr{N}_{1}(\mathscr{K} a)\right)^{p \alpha} d \mu \leq C
$$

for every p-atom $a$.

Proof. Apply Corollary 3.2 to the measure $\nu$ defined on $X^{+}$by $\nu(E)=\sigma(E \cap$ $(X \times\{0\}))$. Then

$$
\begin{aligned}
\int_{X^{+}}\left(\mathscr{N}_{1}(\mathscr{K} a)\right)^{p \alpha} d \mu & =\int_{X^{+}}\left(\left(\mathscr{N}_{1}(\mathscr{K} a)\right)^{p}\right)^{\alpha} d \mu \\
& \leq C \int_{X^{+}}\left(\mathscr{N}_{C_{\rho}}(\mathscr{K} a)\right)^{p} d \nu \leq C^{\prime}
\end{aligned}
$$

by Theorem 4.3.

Corollary 4.5. Let $M$ and $m$ be positive constants, let $M /(M+m)<p \leq 1$, and let $p \leq q<\infty$. Suppose that $\sup _{k} C_{k} 2^{-k M}<\infty$, that $\mu$ and $\gamma$ are Borel measures on $X$ and $\mathbf{R}^{+}$respectively, and that $(\mu(B))^{p / q} \gamma\left(I_{B}\right) \leq \sigma(B)$ for every quasi-ball $B$. Then there is a constant $C=C\left(C_{\rho}, M, m, p, q\right)$ such that

$$
\int_{\mathbf{R}^{+}}\left(\int_{X}\left(\mathscr{N}_{1}(\mathscr{K} a)\right)^{q} d \mu\right)^{p / q} d \gamma \leq C
$$

for every p-atom $a$.

The proof uses Corollary 3.3 and is similar to that of Corollary 4.4.

Theorem 4.2(a) and Corollary 4.4 extend Duren's result, and Theorem 4.2(b) and Corollary 4.5 extend the results of Flett and Hardy-Littlewood mentioned in the introduction.

Theorem 4.6. Let $1<p<\infty, \alpha, \kappa \geq 1$, and $\beta>0$. Suppose that $\mu, \lambda$, and $\gamma$ are Borel measures on $X, \mathbf{R}^{+}$, and $\mathbf{R}^{+}$respectively and that

$$
(\mu(B(x, r)))^{\beta} \lambda[r, 2 r] \leq C(\sigma(B(x, r)))^{\alpha \beta}(\gamma[r / 8, r / 4])^{\kappa}
$$

for every $x \in X$ and $r>0$. Then there is a constant

$$
C^{\prime}=C^{\prime}\left(C, C_{\rho}, C_{\sigma}, p, \alpha, \beta\right)
$$

such that

$$
\int_{\mathbf{R}^{+}}\left(\int_{X} F^{\alpha p} d \mu\right)^{\beta} d \lambda \leq A_{F}^{\alpha \beta p} C^{\prime}\left[\int_{\mathbf{R}^{+}}\left(\int_{X} F^{p} d \sigma\right)^{\alpha \beta / \kappa} d \gamma\right]^{\kappa}
$$

for every Borel measurable function $F \in \mathrm{BR}$. 
Proof. We have

$$
\begin{aligned}
& \int_{\mathbf{R}^{+}}\left(\int_{X} F^{\alpha p} d \mu\right)^{\beta} d \lambda \\
& \leq A_{F}^{\alpha \beta p} C \int_{\mathbf{R}^{+}}\left(\int_{X}\left(\mathscr{H} F_{r / 2}(x, r / 2)\right)^{\alpha p} d \mu(x)\right)^{\beta} d \lambda(r) \text { by Lemma 2.1(e) } \\
& \leq A_{F}^{\alpha \beta p} C\left[\int_{\mathbf{R}^{+}}\left(\int_{X}\left(\mathscr{N}_{C_{\rho}^{2}} \mathscr{H} F_{r / 2}(x, r / 2)\right)^{p} d \sigma(x)\right)^{\alpha \beta / \kappa} d \gamma(r)\right]^{\kappa} \\
& \text { by Theorem } 3.4 \\
& \leq A_{F}^{\alpha \beta p} C\left[\int_{\mathbf{R}^{+}}\left(\int_{X}\left(\mathscr{H} F_{r / 2}(x, r / 2)\right)^{p} d \sigma(x)\right)^{\alpha \beta / \kappa} d \gamma(r)\right]^{\kappa} \\
& \leq A_{F}^{\alpha \beta p} C\left[\int_{\mathbf{R}^{+}}\left(\int_{X}\left(\mathscr{M} F_{r / 2}(x)\right)^{p} d \sigma(x)\right)^{\alpha \beta / \kappa} d \gamma(r)\right]^{\kappa} \\
& \leq A_{F}^{\alpha \beta p} C\left[\int_{\mathbf{R}^{+}}\left(\int_{X} F_{r / 2}^{p} d \sigma\right)^{\alpha \beta / \kappa} d \gamma\right]^{\kappa} \text { since } \mathscr{M} \text { is strong type }(p, p) \\
& \leq A_{F}^{\alpha \beta p} C\left[\int_{\mathbf{R}^{+}}\left(\int_{X} F^{p} d \sigma\right)^{\alpha \beta / \kappa} d \gamma\right]^{\kappa} \quad \text { since } F \in \mathrm{BR} .
\end{aligned}
$$

This completes the proof.

\section{EXAMPLES}

We give several examples of spaces of homogeneous type and of interesting kernels associated with them, and make the connections with the results obtained earlier.

Example 1a. Here $X=\mathbf{R}^{d}, \rho$ is Euclidean distance, and $\sigma$ is Lebesgue measure. Then $C_{\rho}=1$ and $C_{k}=2^{k d}$. Thus if $N>d$, then $\Phi(t)=\left(1+t^{2}\right)^{-N / 2}$ is admissible and satisfies the hypotheses of Lemma 2.1 and Theorems 4.1 and 4.2. In particular, if $N=d+1$, then $K_{t}(x, y)=P_{t}(x, y)=c_{d} t\left(t^{2}+|x-y|^{2}\right)^{-(d+1) / 2}$, which is the Poisson kernel for $\mathbf{R}^{d}$. This kernel satisfies the hypothesis of Theorem 4.3 with $M=d+1$ and $m=1$. Since $P|f| \in \mathrm{BR}, \mathscr{N}_{\alpha}(P f)(x, 0) \leq$ $C(P|f|)^{*}(x, 0) \leq C \mathscr{M} f(x)$, so $\mathscr{N}_{\alpha}(P f)$ is $L^{2}$-bounded.

Example 1b. Here again, $X=\mathbf{R}^{d}$ and $\sigma$ is Lebesgue measure, but now $\rho$ is given by $\rho(x, y)=|x-y|^{2}$. Then $\Phi(t)=e^{-t}$ is admissible and is dominated by $(1+s)^{-N}$ for any $N$. The resulting kernel $K_{t}$ is the reproducing kernel for solutions of the heat equation in $\mathbf{R}_{+}^{d+1}$ and it satisfies the hypothesis of Theorem 4.3 with any $M \geq d$ and $m=1 / 2$. Since $|\mathscr{K} f| \leq C P|f|, \mathscr{N}_{\alpha}(\mathscr{K} f)$ is $L^{2}$-bounded.

A good reference for these kernels is [SW].

Example 2. Here $X$ is the unit sphere in $\mathbf{R}^{d}, \rho$ is Euclidean distance, and $\sigma$ is rotation invariant measure. Then $\sigma(B(x, r)) \sim r^{d-1}$ and $C_{k} \sim 2^{k(d-1)}$. Thus if $N>d-1$, then $\Phi(t)=(1+t)^{-N}$ is admissible and satisfies the 
hypotheses of Lemma 2.1. In particular, if $N=d$, then $K_{t}(x, y) \sim P_{r}(x, y)=$ $c_{d}\left(1-r^{2}\right)|r x-y|^{-d}$ where $r=1-t$, which is the Poisson kernel for $X$. It satisfies the hypotheses of Theorem 4.3 with $M=d$ and $m=1$. The $L^{2}$ boundedness of $\mathscr{N}_{\alpha}(P f)$ follows as before. For details, see [SW].

Example 3. Here $X$ is the unit sphere in $\mathbf{C}^{d}, \rho$ is the nonisotropic metric defined by $\rho(x, y)=|1-\langle x, y\rangle|^{1 / 2}$ (where $\langle x, y\rangle=\sum x_{k} \bar{y}_{k}$ ), and $\sigma$ is rotation invariant measure. Then $\sigma(B(x, r)) \sim r^{2 d}$ and $C_{k} \sim 2^{2 k d}$. Thus if $N>2 d$, then $\Phi(t)=(1+t)^{-N}$ is admissible and satisfies the hypotheses of Lemma 2.1. In particular, if $N=4 d$, then $K_{t}(x, y) \sim P_{r}(x, y)=$ $c_{d}\left(1-r^{2}\right)^{d}|1-r\langle x, y\rangle|^{-2 d}$ where $r^{2}+t^{2}=1$, which is the invariant Poisson kernel. It satisfies the hypothesis of Theorem 4.3 with $M=4 d+1$ and $m=1$. The $L^{2}$-boundedness of $\mathscr{N}_{\alpha}(P f)$ follows as before.

The kernel $C_{t}(x, y)=c_{d}(1-r\langle x, y\rangle)^{-d}$ is the Cauchy kernel. It is complexvalued, and $\left|C_{t}(x, \cdot)\right|$ is not uniformly integrable, but the hypotheses of Theorem 4.3 are satisfied with $M=2 d+1$ and $m=1$; the proof is very similar to that of [R, Lemma 6.1.1]. The $L^{2}$-boundedness of $\mathscr{N}_{\alpha}(\mathscr{C} f)$ is shown in [R, Theorem 5.6.9].

Example 4 (a Heisenberg group). Here $X=\mathbf{R} \times \mathbf{C}^{d-1}$, and $\sigma$ is Lebesgue measure on $\mathbf{R}^{2 d-1}$. If we write $z=(x, \xi)$ and $w=(y, \zeta)$ and let $z \circ w=$ $(x+y+2 \mathfrak{I}\langle\xi, \zeta\rangle, \xi+\zeta)$, then $(X, \circ)$ is a group, and if $\gamma(z)=\left(|x|^{2}+|\xi|^{4}\right)^{1 / 2}$ and $\rho(z, w)=\gamma\left(z \circ w^{-1}\right)$, then $\rho$ is a quasi-metric that is invariant under the group action. Then $\sigma(B(x, r)) \sim r^{d}$ and $C_{k} \sim 2^{k d}$. Thus if $N>d$, then $\Phi(t)=(1+t)^{-N}$ is admissible and satisfies the hypotheses of Lemma 2.1. In particular, if $N=2 d$, then $K_{t}(z, w) \sim P_{t}(z, w)$ where $P_{t}(z, 0)=$ $c_{d} t^{d}\left(|x|^{2}+\left(t+|\xi|^{2}\right)^{2}\right)^{-d}$ and $P_{t}$ is invariant under the group action. This is the Poisson kernel for $X$. The hypotheses of Theorem 4.3 are satisfied with $M=2 d$ and $m=1 / 2$. The $L^{2}$-boundedness of $\mathscr{N}_{\alpha}(P f)$ follows as in Example 2.

The Cauchy-Szegö kernel for $X$ is given by

$$
C_{t}(z, w)=c_{d}\left(t+|\xi-\zeta|^{2}-i(x-y-2 \mathfrak{I}\langle\xi, \zeta\rangle)\right)^{-d}
$$

It satisfies the hypotheses of Theorem 4.3 with $M=d$ and $m=1 / 2$. The proofs of these facts are similar to those in Example 3. A good reference is [KV].

Example 5. Here $X$ is the $p$-adic field, $\rho$ is the usual metric on $X$, and $\sigma$ is Haar measure on $X$ regarded as a locally compact group under addition. See [T] for details. Then $\sigma\left(B\left(x, 2^{-k}\right)\right)=2^{-k}$. Thus $\Phi(x)=\chi_{[0,1]}(x)$ is admissible and the resulting kernel is the Poisson kernel $P_{k}(x, y)=2^{-k} \chi_{B\left(x, 2^{-k}\right)}(y)$.

Example 6. Here $X=[0, \pi], \rho$ is the usual metric on $X$, and $\sigma$ is given for $\lambda>0$ by $d \sigma(x)=\sin ^{2 \lambda} x d x$. See [MS] for details. This space is associated with the ultraspherical harmonics. The Poisson kernel is

$$
\operatorname{Pr}(\theta, \phi)=\pi^{-1} \lambda\left(1-r^{2}\right) \int_{0}^{\pi} \sin ^{2 \lambda-1} t\left[1+r^{2}-2 r(\cos \theta \cos \phi+\sin \theta \sin \phi \cos t)\right]^{-\lambda-1} d t .
$$

This kernel is not generated by a function $\Phi$ as in the other examples, but the proof in [MS] that shows that the operator $\mathscr{K}$ determined by $P_{r}$ is dominated 
by the Hardy-Littlewood maximal operator may be slightly altered to show that $\mathscr{K}$ is dominated by the Hörmander maximal operator $\mathscr{H}$.

Muckenhoupt and Stein [MS] also study the Fourier-Bessel expansions for functions on $\mathbf{R}$. There is a Poisson kernel in this case that is similar to the one described above and the same remarks pertain to it.

\section{The APPLiCATIONS AND THE EXAMPLES}

The results that have been obtained have dealt with general spaces of homogeneous type, and one might ask if they are sharp enough to yield known results when they are applied to the examples in $\S 5$. We show that they are in the following settings.

In Example 2, $X$ is the unit sphere in $\mathbf{R}^{d}, \rho$ is Euclidean distance, and $\sigma$ is rotation invariant measure. The Poisson kernel is

$$
P_{r}(x, y)=c\left(1-r^{2}\right) /|r x-y|^{d} .
$$

If $f \in L^{2}(\sigma)$ and $0<r<1$, then

$$
P f(x, r)=\sum_{n} \sum_{k} c_{n k} r^{n} Y_{n k}(x)
$$

where $\left\{Y_{n k} \mid n=1,2,3, \ldots, k=1,2,3, \ldots, \alpha_{n}\right\}$ is an orthonormal sequence in $L^{2}(\sigma)$ (the spherical harmonics) and $c_{n k}=\int_{X} f Y_{n k} d \sigma$. So

$$
\int_{X}|P f(x, r)|^{2} d \sigma(x)=\sum_{n} \sum_{k}\left|c_{n k}\right|^{2} r^{2 n} \text {. }
$$

(See [SW].)

Theorem 6.1. In the setting of Example 2, let $d /(d+1)<p \leq 1$ and let $\beta=(d-1)(1-p / 2)$. Then there is a constant $C=C(d, p)$ such that

$$
\sum_{N=1}^{\infty} 2^{-N \beta}\left(\sum_{n=2^{N}}^{2^{N+1}} \sum_{k=1}^{\alpha_{n}}\left|c_{n k}\right|^{2}\right)^{p / 2} \leq C
$$

for every p-atom $a$.

Proof. Apply Corollary 4.5 with $\mu=\sigma, q=2$, and $d \gamma(t)=t^{\beta-1} d t$. Then

$$
\int_{0}^{1}\left(\int_{X}|P a(x, r)|^{2} d \sigma(x)\right)^{p / 2} d \gamma(r) \leq C .
$$

The conclusion follows immediately from this and the remark preceding the statement of the theorem.

An immediate corollary of this result is a sufficient condition for a sequence to multiply $H_{\text {atom }}^{p}$ into $l^{p}$.

Corollary 6.2. In the setting of Example 2 , let $d /(d+1)<p \leq 1$. Suppose that there is a constant $C$ and a doubly indexed set of constants $\left\{\bar{w}_{n k}\right\}_{n, k}$ such that

$$
\sum_{n=2^{N}}^{2^{N+1}} \sum_{k=1}^{\alpha_{n}}\left|w_{n k}\right|^{p /(1-p / 2)} \leq C 2^{-N(d-1)}
$$


for every $N \in \mathbf{N}$. Then there is a constant $C^{\prime}=C^{\prime}(d, p)$ such that

$$
\sum_{n} \sum_{k}\left|w_{n k} c_{n k}\right|^{p}<C^{\prime}
$$

for every p-atom a.

Proof. By Hölder's inequality,

$$
\sum_{n} \sum_{k}\left|w_{n k} c_{n k}\right|^{p} \leq \sum_{N=1}^{\infty}\left(\sum_{n=2^{N}}^{2^{N+1}} \sum_{k=1}^{\alpha_{n}}\left|c_{n k}\right|^{2}\right)^{p / 2}\left(\sum_{n=2^{N}}^{2^{N+1}} \sum_{k=1}^{\alpha_{n}}\left|w_{n k}\right|^{p /(1-p / 2)}\right)^{1-p / 2} .
$$

The proof is completed by applying Theorem 6.1.

In particular, when $p=1$, this implies that

$$
\sum_{n} \sum_{k}\left|c_{n k}\right| / n^{d-1} \leq C
$$

for every 1-atom, and consequently that this is true for every function in $H_{\text {atom }}^{1}$. This is an extension of Hardy's inequality [HL1].

Similar considerations apply to the next example.

In Example 3, $X$ is the unit sphere in $\mathbf{C}^{d}, \rho$ is the nonisotropic metric, and $\sigma$ is rotation invariant measure. If $z \in X$ and $r>0$, then $\mathscr{C} a(z, r)=$ $\sum_{\alpha} c_{\alpha} r^{|\alpha|} z^{\alpha} /\left\|z^{\alpha}\right\|_{2}^{2}$ for any $p$-atom $a$, where $c_{\alpha}=\int_{X} a(w) \bar{w}^{\alpha} d \sigma(w)$ for any multi-index $\alpha$. Now, $\left\{z^{\alpha} /\left\|z^{\alpha}\right\|_{2}\right\}$ is an orthonormal sequence, so if $b_{\alpha}=$ $c_{\alpha} /\left\|z^{\alpha}\right\|_{2}$, then $\sum_{\alpha}\left|b_{\alpha}\right|^{2} r^{2|\alpha|}=\int_{X}|\mathscr{C} a(x, r)|^{2} d \sigma(x)$.

The same ideas that were involved in the proof of Theorem 6.1 and Corollary 6.2 prove the following results.

Theorem 6.3. In the setting of Example 3 , let $(2 d+1) /(2 d+2)<p \leq 1$ and $\beta=d(1-p / 2)$. Then there is a constant $C=C(d, p)$ such that

$$
\sum_{N=1}^{\infty} 2^{-N \beta}\left(\sum_{2^{N} \leq|\alpha| \leq 2^{N+1}}\left|b_{\alpha}\right|^{2}\right)^{p / 2} \leq C
$$

for every p-atom $a$.

Thus if

$$
\sum_{2^{N} \leq|\alpha| \leq 2^{N+1}}\left|w_{\alpha}\right|^{p /(1-p / 2)} \leq C 2^{-N d},
$$

then $\sum_{\alpha}\left|w_{\alpha} b_{\alpha}\right|^{p}<\infty$ for every $p$-atom $a$. Again, when $p=1$, this yields an extension of Hardy's inequality.

\section{NeCESSARY CONDITIONS FOR CARLESON MEASURES}

It is natural to inquire if the conditions that were sufficient in the Carleson measure theorems are also necessary. The next theorem, a converse to Theorem 4.1 , is typical of what may be proved along these lines.

Theorem 7.1. Let $0<p<\infty$. Suppose that $\mu$ and $\nu$ are Borel measures on $X^{+}$such that

$$
0<\nu(T(\widetilde{B})) \leq C \nu(T(B))
$$


for every quasi-ball $B$ and

$$
\int_{X^{+}}\left(\mathscr{H}_{\nu} F\right)^{p} d \mu \leq \int_{X^{+}} F^{p} d \nu
$$

for every bounded measurable $F \in \mathrm{BR}$. Then there is constant $C^{\prime}=C^{\prime}(C, p)$ so that

$$
\mu(T(B)) \leq C^{\prime} \nu(T(B))
$$

for every quasi-ball $B$.

Proof. Fix $y \in X$ and $s>0$ and define $F(x, r)=(s+r+\rho(x, y))^{-M}$ where $M$ will be chosen later. If $(x, t) \in T(B(y, s))$ then

$$
\mathscr{H}_{\nu} F(z, t) \geq \frac{\int_{T(B(y, s))} F d \nu}{\nu(T(B(y, s)))} \geq 2^{-M^{-M}}
$$

and for large enough $M$,

$$
\begin{aligned}
\int_{X^{+}} F^{p} d \nu & =\int_{T(B(y, s))} F^{p} d \nu+\sum_{k=0}^{\infty} \int_{T\left(B\left(y, 2^{k+1} s\right)\right) \backslash T\left(B\left(y, 2^{k} s\right)\right)} F^{p} d \nu \\
& \leq C s^{-M p} \nu(T(B(y, s))) .
\end{aligned}
$$

Combining these two facts completes the proof.

There are similar converse results for Corollaries 3.2 and 3.3 and Theorems 3.4 and 4.2. These may be obtained using the same ideas and the same functions that were used in Theorem 7.1.

In the proof of Theorem 7.1 it was possible to use a smaller collection of test functions than the set of functions for which Theorem 4.1 holds true. In specific examples, it may not be immediately clear which test functions to choose. For example, the analytic functions are an adequate class of test functions in the original Carleson measure theorem [Car], and it is reasonable to expect that they will also be adequate if $X$ is the unit sphere in $\mathbf{C}^{d}$. If $X$ is formed into a space of homogeneous type as in Example 3 of $\S 5$, then Hörmander [Hö] showed that the analytic functions are indeed an adequate test class, but he also showed that if $X$ is made into a space of homogeneous type as in Example 2 of $\S 5$, then they are not, but the harmonic functions are. Thus, determining the right collection to serve as a test class poses some interesting problems. The next theorem is one example of what may be done in this direction.

Theorem 7.2. Let $\alpha>0, \beta>0$, and $c>-1$. Suppose that $\mu$ and $\gamma$ are Borel measures on $\mathbf{R}^{d}$ and $\mathbf{R}^{+}$respectively and that

$$
\begin{aligned}
\int_{\mathbf{R}^{+}} & \left(\int_{\mathbf{R}^{d}}|F(x, r)|^{\alpha} d \mu(x)\right)^{\beta} d \gamma(r) \\
& \leq \int_{\mathbf{R}^{+}}\left(\int_{\mathbf{R}^{d}}|F(x, r)|^{2} d x\right)^{\alpha \beta / 2} r^{c} d \gamma(r)
\end{aligned}
$$

for every harmonic function $F$. Then there is a constant $C=C(d, \alpha, \beta, c)$ such that

$$
(\mu(B(x, r)))^{\beta} \gamma([0, r]) \leq C r^{(\alpha \beta d / 2)+c+1}
$$

for every $x \in \mathbf{R}^{d}$ and $r>0$. 
Proof. Fix $\delta>0$ and for $k \in \mathbf{Z}$ let $Q_{k}=\left\{x \in \mathbf{R}^{d}|| x_{1}-2 k \delta|<\delta,| x_{2} \mid<\right.$ $\left.\delta, \ldots,\left|x_{d}\right|<\delta\right\}$. Then $\left\{Q_{k}\right\}$ is a disjoint family of cubes of side length $\delta$, and

$$
\widehat{\chi}_{Q_{k}}(x)=C e^{-2 i k \delta x_{1}} \frac{\sin \delta x_{1}}{x_{1}} \ldots \frac{\sin \delta x_{d}}{x_{d}} \equiv C e^{-2 i k \delta x_{1}} H(x),
$$

so if $\phi(x)=\sum_{k=0}^{p}\left(\begin{array}{c}p \\ k\end{array}\right)(-1)^{k} \chi Q_{Q_{k}}(x)$, then $\hat{\phi}(x)=C H(x)\left(1-e^{-2 i k \delta x_{1}}\right)^{p}$. Consequently, if $F(x, r)=P \phi(x, r)$, then $F$ is harmonic and

$$
\begin{aligned}
\int_{\mathbf{R}^{d}}|F(x, r)|^{2} d x & =C \int_{\mathbf{R}^{d}} e^{-2 r|x|}|H(x)|^{2}\left|1-e^{2 i \delta x_{1}}\right|^{2 p} d x \\
& =C \int_{\mathbf{R}^{d}} e^{-2 r|x|}|H(x)|^{2}\left|\sin \delta x_{1}\right|^{2 p} d x .
\end{aligned}
$$

Now,

$$
\begin{gathered}
\int_{\mathbf{R}^{d}} e^{-2 r|x|}|H(x)|^{2}\left|\sin \delta x_{1}\right|^{2 p} d x \leq C \delta^{d}, \\
\int_{\{|x| \leq 1 / \delta\}} e^{-2 r|x|}|H(x)|^{2}\left|\sin \delta x_{1}\right|^{2 p} d x \\
\leq C\left(\int_{0}^{1 / \delta} e^{-2 r x d^{-1 / 2}} \frac{|\sin \delta x|^{2+2 p}}{x^{2}} d x\right) \\
\cdot\left(\int_{0}^{1 / \delta} e^{-2 r x d^{-1 / 2}} \frac{|\sin \delta x|^{2}}{x^{2}} d x\right)^{d-1} \\
\leq \begin{cases}C \delta^{2 d+2 p} / r^{d+2 p} & \text { if } \delta \leq r \\
C \delta^{d} & \text { if } \delta \geq r,\end{cases}
\end{gathered}
$$

and

$$
\int_{\{|x| \geq 1 / \delta\}} e^{-2 r|x|}|H(x)|^{2}\left|\sin \delta x_{1}\right|^{2 p} d x \leq C \delta^{d} e^{-2 r / \delta}
$$

Let

$$
\begin{aligned}
I= & \int_{0}^{\infty}\left(\int_{\mathbf{R}^{d}}|F|^{2} d x\right)^{\alpha \beta / 2} r^{c} d r \\
\leq & \int_{0}^{\delta}\left(\int_{\mathbf{R}^{d}}|F|^{2} d x\right)^{\alpha \beta / 2} r^{c} d r+C \int_{\delta}^{\infty}\left(\int_{|x| \leq 1 / \delta}|F|^{2} d x\right)^{\alpha \beta / 2} r^{c} d r \\
& +C \int_{\delta}^{\infty}\left(\int_{|x| \geq 1 / \delta}|F|^{2} d x\right)^{\alpha \beta / 2} r^{c} d r \\
= & I_{1}+I_{2}+I_{3} .
\end{aligned}
$$

Then $I_{1} \leq C \delta^{\alpha \beta d / 2+c+1}$ by (1), $I_{3} \leq C \delta^{\alpha \beta d / 2} \int_{\delta}^{\infty} e^{-\alpha \beta r / \delta} r^{c} d r=C \delta^{\alpha \beta d / 2+c+1}$ by (3), and $I_{2} \leq C \delta^{\alpha \beta(2 p+2 d) / 2} \int_{\delta}^{\infty} r^{c-\alpha \beta(d+2 p) / 2} d r=C \delta^{\alpha \beta d / 2+c+1}$ by (2) if $p$ is chosen large enough so that $\alpha \beta(d+2 p) / 2>c+1$. Thus $I \leq C \delta^{\alpha \beta d / 2+c+1}$. 
Next we claim that there is a constant $\lambda$ such that $|F(x, r)| \geq 1 / 2$ if $|x| \leq \lambda \delta$ and $r \leq \lambda \delta$. From this and the hypothesis of the theorem it follows that

$$
\gamma([0, \lambda \delta))(\mu(B(0, \lambda \delta)))^{\alpha \beta} \leq C \delta^{\alpha \beta d / 2+c+1} .
$$

To prove the claim, note that

$$
\begin{aligned}
1-F(x, r) & =1-\int_{Q_{0}} P_{r}(x, y) d y-\sum_{k=1}^{p}\left(\begin{array}{l}
p \\
k
\end{array}\right)(-1)^{k} \int_{Q_{k}} P_{r}(x, y) d y \\
& =\int_{\mathbf{R}^{d} \backslash Q_{0}} P_{r}(x, y) d y-\sum_{k=1}^{p}\left(\begin{array}{l}
p \\
k
\end{array}\right)(-1)^{k} \int_{Q_{k}} P_{r}(x, y) d y .
\end{aligned}
$$

But if $k \geq 1$, then $Q_{k} \subset \mathbf{R}^{d} \backslash Q_{0}$, so $|1-F(x, r)| \leq 2^{p} \int_{\mathbf{R}^{d} \backslash Q_{0}} P_{r}(x, y) d y$. Now it easy to see that if $\delta>0, \lambda \leq 1 / 2,|x| \leq \lambda \delta$, and $|r| \leq \lambda \delta$, then $\int_{|y| \geq \delta} P_{r}(x, y) d y \leq C_{d} \lambda$, so $|1-F(x, r)| \leq 1 / 2$ for sufficiently small $\lambda$, and hence $|F(x, r)| \geq 1 / 2$ if $|x| \leq \lambda \delta$ and $r \leq \lambda \delta$. This completes the proof of Theorem 7.2.

\section{REFERENCES}

[AB] E. Amar and A. Bonami, Mesures de Carleson d'ordre $\alpha$ et solutions au bord de l'equation $\bar{\partial}$, Bull. Soc. Math. France 107 (1979), 23-48.

[BM] R. Bañuelos and C. N. Moore, Laws of the iterated logarithm, sharp good- $\lambda$ inequalities and $L^{p}$-estimates for caloric and harmonic functions, Indiana Math. J. 38 (1989), 315-344.

[B] D. Békollé, Inégalités à poids pour le projecteur de Bergman dans la boule unité de $\mathbf{C}^{n}$, Studia Math. 71 (1982), 305-323.

[BP] A. Benedek and R. Panzone, The spaces $L^{p}$, with mixed norm, Duke Math. J. 28 (1961), 301-324.

[Cal] A. P. Calderón, Inequalities for the maximal function relative to a metric, Studia Math. 57 (1976), 297-306.

[Car] L. Carleson, Interpolation by bounded analytic functions and the corona problem, Ann. of Math. (2) 76 (1962), 547-559.

[CiWo] J. A. Cima and W. R. Wogen, A Carleson measure theorem for the Bergman space on the ball, J. Operator Theory 7 (1982), 157-165.

[CW1] R. Coifman and G. Weiss, Analyse harmonique non-commutative sur certains espaces homogènes, Lecture Notes in Math., vol. 242, Springer-Verlag, 1971.

[CW2] _ Extensions of Hardy spaces and their use in analysis, Bull. Amer. Math. Soc. 83 (1977), 569-645.

[D] P. Duren, Extension of a theorem of Carleson, Bull. Amer. Math. Soc. 75 (1969), 143-146.

[FS] R. Fefferman and E. M. Stein, Some maximal inequalities, Amer. J. Math. 93 (1971), 107-115.

[Fl] T. M. Flett, On the rate of growth of mean values of holomorphic and harmonic functions, Proc. London Math. Soc. (3) 20 (1970), 749-768.

[Fu] B. Fuglede, On the theory of potentials in locally compact spaces, Acta Math. 103 (1960), 139-215.

[Gr] I. Graham, The radial derivative, fractional integrals, and comparative growth of means of holomorphic functions on the unit ball in $\mathbf{C}^{n}$, Ann. of Math. Stud., no. 100, Princeton Univ. Press, 1981, pp. 171-178.

[HL1] G. H. Hardy and J. E. Littlewood, Some new properties of Fourier constants, Math. Ann. 97 (1926), 159-209.

[HL2] _ Theorems concerning mean values of analytic or harmonic functions, Quart. J. Math. 12 (1941), 221-256. 
[Ha] W. Hastings, A Carleson measure theorem for Bergman spaces, Proc. Amer. Math. Soc. 52 (1975), 237-241.

[Hö] L. Hörmander, $L^{p}$ estimates for (pluri-)subharmonic functions, Math. Scand. 20 (1967), 65-78.

[KV] A. Korányi and S. Vági, Singular integrals on homogeneous spaces and some problems of classical analysis, Ann. Scuola Norm. Sup. Pisa Cl. Sci. 25 (1971), 575-648.

[L] D. Luecking, A technique for characterizing Carleson measures on Bergman spaces, Proc. Amer. Math. Soc. 87 (1983), 656-660.

[MS] B. Muckenhoupt and E. M. Stein, Classical expansions and their relation to conjugate harmonic functions, Trans. Amer. Math. Soc. 118 (1965), 17-92.

[R] W. Rudin, Function theory in the unit ball of $\mathbf{C}^{n}$, Springer-Verlag, 1980.

[RT] F. R. Ruiz and J. L. Torrea, Vector-valued Calderón-Zygmund theory and Carleson measures on spaces of homogeneous nature, Studia Math. 88 (1988), 221-243.

[S] E. M. Stein, Singular integrals and differentiability properties of functions, Princeton Univ. Press, 1970.

[SW] E. M. Stein and G. Weiss, Introduction to Fourier analysis on Euclidean spaces, Princeton Univ. Press, 1971.

[T] M. H. Taibleson, Fourier analysis on local fields, Math. Notes 15, Princeton Univ. Press, 1975.

Department of Mathematics, Rhodes College, Memphis, Tennessee, 38112-1690

E-mail address: gadbois@rhodes.bitnet

Department of Mathematics, Michigan State University, East Lansing, Michigan, 48824

E-mail address: 21144 $\mathrm{wts}$ @msu.bitnet 